Check for updates

Cite this: Chem. Sci., 2018, 9, 6796

๑ All publication charges for this article have been paid for by the Royal Society of Chemistry

Received 15th May 2018

Accepted 12th July 2018

DOI: $10.1039 / \mathrm{c} 8 \mathrm{sc0} 02151 \mathrm{a}$

rsc.li/chemical-science

\section{Enhancement of triplet-sensitized upconversion in rigid polymers via singlet exciton sink approach $\dagger$}

\author{
Steponas Raišys, ${ }^{a}$ Saulius Juršènas, ${ }^{a}$ Yoan C. Simon, (D) ${ }^{\text {bc }}$ Christoph Weder (D) ${ }^{\text {b }}$ \\ and Karolis Kazlauskas (D) *a
}

\begin{abstract}
To increase the practical usefulness of solid-state sensitized upconversion (UC) materials as components of solar energy harvesting systems, it is important to identify and suppress loss mechanisms, and increase the UC quantum yield $\left(\Phi_{\cup C}\right)$. Here we focus on a benchmark UC system consisting of the emitter 9,10diphenylanthracene (DPA) and the sensitizer platinum octaethylporphyrin (PtOEP) in a rigid poly(methyl methacrylate) (PMMA) matrix, and show that one of the major losses originates from Förster resonant energy transfer (FRET) from DPA back to PtOEP. Even though DPA emission lies within the PtOEP transparency window, the quantitative assessment of singlet exciton diffusion for samples with a high DPA content evidences that long-range FRET results in effective exciton trapping by PtOEP. A dramatic factor-of- 6 reduction of the DPA emission quantum yield occurs even at PtOEP concentrations as low as 0.05 wt\%. To alleviate this problem, we demonstrate a new concept based on the introduction of highly emissive sink sites to trap the singlet excitons produced upon annihilation prior to their quenching by the sensitizer. For DPA/PtOEP blends in PMMA, 1,6-bis-[2,5-di(dodecyloxyphenyl)ethynyl]pyrene is shown to be a useful sink, which results in 1.5 -fold increase of the $\Phi_{\cup c}$. A maximum $\Phi_{\cup \mathrm{C}}$ of $2.7 \%$ was achieved, which is among the highest reported values for rigid sensitized UC polymers.
\end{abstract}

\section{Introduction}

Light upconversion (UC) relying on triplet-triplet annihilation (TTA) in organic molecules after energy transfer from a sensitizer is a useful process in the context of bioimaging, ${ }^{1}$ photocatalysis, ${ }^{2}$ oxygen sensing, ${ }^{3}$ drug delivery, ${ }^{4,5}$ and in particular solar energy harvesting..$^{6-9}$ Namely, unexploited sub-bandgap photons are harvested and converted into a radiation of higher energy within the absorption region of a solar cell. ${ }^{10} \mathrm{UC}$ proceeds via the triplet manifold of emitter molecules, which usually cannot be directly excited, and must therefore be populated via sensitization. The sensitizer serves a dual purpose: (i) it ensures the absorption of incident light as well as (ii) the rapid conversion of the photo-excited singlets into triplets via intersystem crossing. These processes are followed by triplet energy transfer to emitter molecules (see ESI Fig. S1† for detailed mechanistic information). When nonradiative decay pathways are unfavored, the population of these long-lived emitter (or annihilator) triplet states increases until TTA

anstitute of Photonics and Nanotechnology, Vilnius University, Sauletekio av. 3, LT-10257 Vilnius, Lithuania. E-mail: karolis.kazlauskas@ff.vu.lt

${ }^{b}$ Adolphe Merkle Institute, University of Fribourg, Chemin des Verdiers 4, CH-170O Fribourg, Switzerland

${ }^{c}$ School of Polymer Science and Engineering, University of Southern Mississippi, 118 College Drive \#5050, Hattiesburg, Mississippi 39406, USA

$\dagger$ Electronic supplementary information (ESI) available. See DOI: $10.1039 / \mathrm{c} 8 \mathrm{sc} 02151 \mathrm{a}$ results in the formation of emitter singlet excited states, which relax radiatively. ${ }^{8}$ This process enables UC under weak $(<10 \mathrm{~mW}$ $\mathrm{cm}^{-2}$ ) and noncoherent irradiation, which is readily available from the sun. ${ }^{11,12}$

The most efficient sensitized TTA-UC attained so far are limited to solutions/liquids $\left(\Phi_{\mathrm{UC}} \approx 30 \%\right),{ }^{13}$ liquid nanodroplets encapsulated within rigid matrices $\left(\Phi_{\mathrm{UC}}=15 \%\right)^{14}$ or 'soft' matrixes, e.g. elastomers, ${ }^{15}$ gels, ${ }^{16,17}$ and other materials ${ }^{18}$ in which the dye molecules enjoy a high degree of rotational and translational motion, and encounter of excited molecular species enable triplet energy transfer and TTA. In polymer matrices, high efficiency is thus readily observed in systems with low glass transition temperature $\left(T_{\mathrm{g}}\right)$, i.e. where $T>T_{\mathrm{g}}$. For practical applications, rigid UC materials (i.e. with high $T_{\mathrm{g}}$ ) are often preferable. In such systems, the positions of sensitizers and emitters are fixed, and TTA depends solely on the triplet exciton diffusion. Regrettably, here $\Phi_{\mathrm{UC}}$ is remarkably low and rarely exceeds $2 \%$, and overcoming this limit proved challenging. ${ }^{19-24}$ Note that the theoretical maximum for TTA-UC process is defined as $50 \%$, since only one photon can be emitted per two absorbed ones. ${ }^{25}$

Although a full understanding of the TTA-UC loss mechanisms is still lacking, the major losses in rigid solids are generally associated with poor triplet exciton diffusion. ${ }^{\mathbf{1 2 , 2 5 - 2 7}}$ Nevertheless, we recently demonstrated that, in melt-processed UC polymer glasses at high emitter loadings ( $>25 \mathrm{wt} \%$ ), exciton diffusion is not the limiting factor. ${ }^{20}$ Instead, diffusion- 
enhanced nonradiative decay of triplet emitter excitons was implicated as the dominant quenching mechanism. Another significant loss channel, which becomes increasingly important at high chromophore loadings (which are needed in rigid matrices to enable exciton diffusion), is related to back-transfer of upconverted emitter singlets to the sensitizer (ESI Fig. S1†). ${ }^{\mathbf{1 0 2 5 , 2 8 - 3 1}}$ These losses are particularly severe in the case of recently introduced sensitizers such as semiconductor nanocrystals, ${ }^{\mathbf{1 0}}$ thermally activated delayed fluorescence compounds, ${ }^{32,33}$ and direct triplet absorbers, ${ }^{34}$ which all feature broad absorption bands and lack a significant window of transparency. ${ }^{25}$ Obviously, long-range Förster resonant energy back-transfer and the diffusion of generated singlet excitons need to be properly managed to avoid diffusion-assisted trapping by the sensitizer and maximize $\Phi_{\mathrm{UC}}$.

To this end, detrimental Förster resonant energy transfer (FRET) from the emitter to the sensitizer was systematically investigated in a rigid poly(methyl methacrylate) (PMMA, $T_{\mathrm{g}} \approx$ $105{ }^{\circ} \mathrm{C}$ ) matrix by evaluating the singlet exciton diffusion in the presence and absence of sensitizer. Platinum octaethylporphyrin (PtOEP) and 9,10-diphenylanthracene (DPA) were chosen as a model sensitizer-emitter pair for this investigation. Upconverting films were melt-processed, ${ }^{35}$ to ensure homogeneous DPA distribution at high concentration and high $\Phi_{\mathrm{UC}}{ }^{20}$ The singlet exciton diffusion was quantified via a time-resolved photoluminescence bulk-quenching technique. ${ }^{36}$ Finally, a singlet exciton sink approach was tested to circumvent the FRET issue and enhance UC efficiency by trapping singlet excitons, at intentionally introduced, highly emissive sink sites, before they reached the sensitizer.

\section{Results and discussion}

\section{Impact of FRET}

Fig. 1a compares the fluorescence quantum yield $\left(\Phi_{\mathrm{FL}}\right)$ of DPA/ PMMA and DPA/PtOEP/PMMA films and demonstrates the fluorescence quenching of DPA as a result of FRET to PtOEP. $\Phi_{\mathrm{FL}}$ was measured by directly exciting DPA at $\lambda_{\mathrm{ex}}=405 \mathrm{~nm}$. The minute PtOEP content in DPA/PtOEP/PMMA films (several hundred times smaller than that of DPA) and the low extinction coefficient of PtOEP at $\lambda_{\text {ex }}=405 \mathrm{~nm}$ ensured that sensitizer absorption was negligible. In the absence of sensitizer, $\Phi_{\mathrm{FL}}$ of DPA was found to be as high as $\sim 88 \%$, decreasing down to $57 \%$ with increasing DPA concentration, due to self-quenching (Fig. 1a). ${ }^{20}$ In the presence of PtOEP, $\Phi_{\mathrm{FL}}$ of DPA was reduced to $\sim 15 \%$, independently of the DPA content. The markedly reduced $\Phi_{\mathrm{FL}}$ of DPA clearly demonstrates considerable fluorescence quenching by the sensitizer, despite a concentration 300800 times smaller than that of DPA (15-40 wt\%). The quenching effect of the sensitizer is also reflected in fluorescence transients of DPA, which show accelerated decay and a significant drop in fluorescence lifetime from $\sim 9$ to 3 ns upon incorporation of PtOEP (ESI Fig. S2 $\dagger$ ). DPA fluorescence quenching is explained by the overlap of the DPA emission and the PtOEP absorption (Fig. 1b). Even though DPA-PtOEP is considered to be a well-matched emitter-sensitizer pair, as DPA emission lies within the PtOEP "transparency window" (i.e. between the higher energy Soret-band and the lower energy Q-band), FRETinduced quenching is nevertheless significant and decreases $\Phi_{\mathrm{FL}}$ by 6 times at a DPA loading of $25 \mathrm{wt} \%$.

Since the presence of the sensitizer, even in low concentration, was found to have a significant impact on the DPA $\Phi_{\mathrm{FL}}$ in DPA/PtOEP/PMMA films, the optical properties were systematically investigated as a function of the PtOEP concentration. Fig. 2 shows the decrease of $\Phi_{\mathrm{FL}}$ and $\Phi_{\mathrm{UC}}$ of DPA/PtOEP/PMMA films with increasing PtOEP concentrations. To map a broad parameter space, the experiments were performed at different DPA concentrations. Pictures of DPA/PtOEP/PMMA films show a visual increase in PtOEP phosphorescence as PtOEP concentration increases from 0.01 to $0.8 \mathrm{wt} \%$ (ESI Fig. S3 $\dagger$ ). For low PtOEP content and therefore lower absorbance at $\lambda_{\mathrm{ex}}=532 \mathrm{~nm}$, the scattered green excitation from the laser is more apparent.

The $\Phi_{\mathrm{FL}}$ data indicate that the extent of fluorescence quenching increases with the PtOEP content, irrespective of DPA concentration. At $c_{\mathrm{PtOEP}}<10^{-3} \mathrm{wt} \%, \Phi_{\mathrm{FL}}$ approached $80 \%$ but dropped by more than 2 orders of magnitude when the
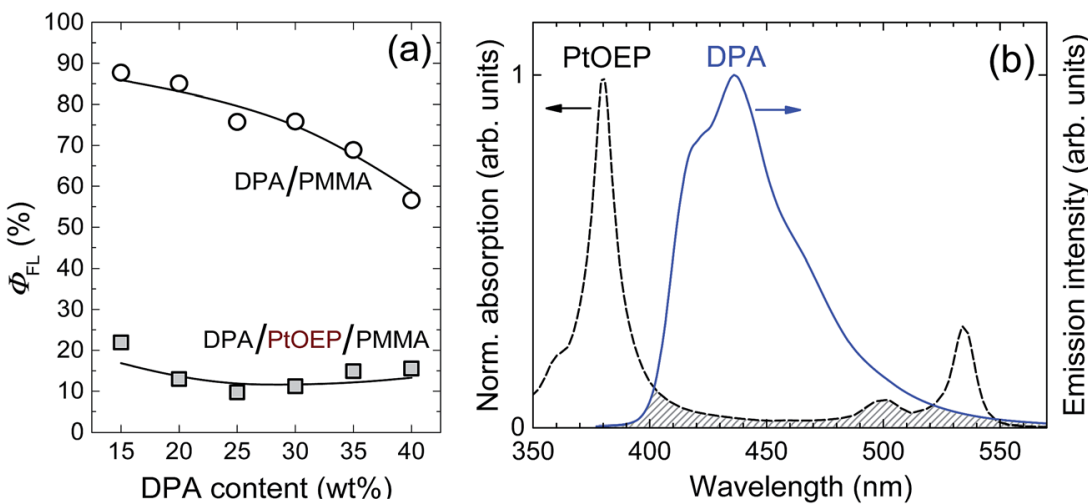

Fig. 1 (a) Fluorescence quantum yield of DPA as a function of its concentration in the PMMA matrix without (circles) and with (squares) PtOEP $\left(c_{\text {PtOEP }}=0.05 \mathrm{wt} \%\right)$. The excitation wavelength $\left(\lambda_{\mathrm{ex}}=405 \mathrm{~nm}\right)$ corresponds to the absorption maximum of DPA. Lines are guides to the eye. (b) Normalized absorption (dashed line) and emission spectra (solid line) of melt-processed PtOEP/PMMA $\left(c_{\text {PtOEP }}=0.05\right.$ wt $\left.\%\right)$ and DPA/PMMA $\left(c_{\text {DPA }}\right.$ $=25 \mathrm{wt} \%$ ) films, respectively. The shaded area indicates spectral overlap. 


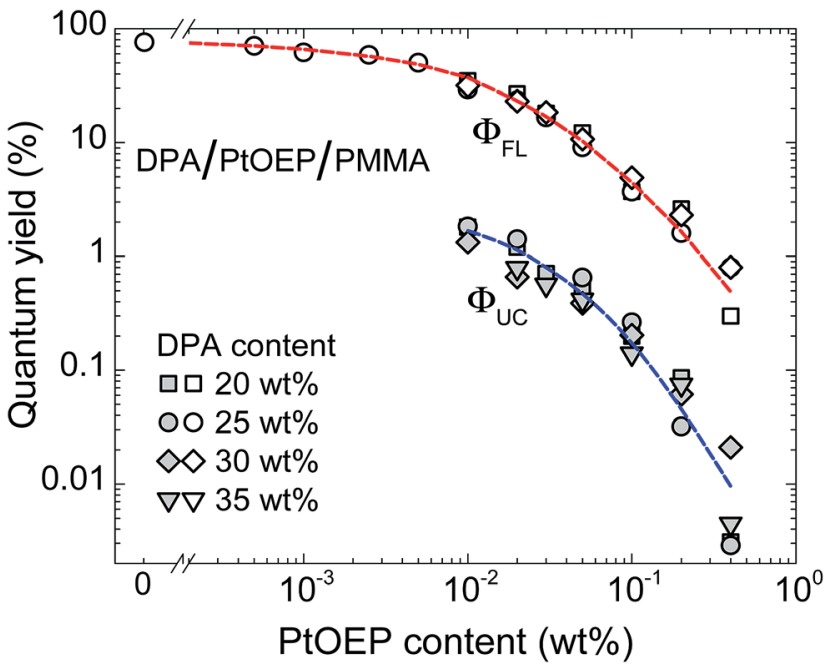

Fig. 2 Fluorescence (open symbols) and UC emission (filled symbols) quantum yield of DPA/PtOEP/PMMA films as a function of PtOEP concentration at different DPA concentrations (20, 25, 30 and $35 \mathrm{wt} \%$ ). The excitation wavelengths for fluorescence and upconversion were $\lambda_{\text {ex, FL }}=405$ and $\lambda_{\text {ex, } \mathrm{UC}}=532 \mathrm{~nm}$, respectively. Lines are guides to the eye.

PtOEP content was increased to above $0.4 \mathrm{wt} \%$. Interestingly, the $\Phi_{\mathrm{UC}}$ follows a similar trend, suggesting that the same emission quenching mechanism is at play, i.e. FRET from DPA to the PtOEP. Note however that a significant $\Phi_{\mathrm{UC}}$ (up to $1.8 \%$ ) could be achieved by reducing the sensitizer concentration to $0.01 \mathrm{wt} \%$. Unfortunately, a further reduction of the PtOEP content led to very low light absorption, and consequently a weak UC signal, making the $\Phi_{\mathrm{UC}}$ measurements unreliable. We stress that in all our experiments both $\Phi_{\mathrm{FL}}$ and $\Phi_{\mathrm{UC}}$ are defined in the same way, i.e. as the number of photons emitted per number of photons absorbed. Therefore, while for fluorescence maximal $\Phi_{\mathrm{FL}}$ is $100 \%$, for UC theoretically attainable maximal $\Phi_{\mathrm{UC}}$ is only $50 \%$ (as TTA-UC process requires two absorbed photons per one emitted). ${ }^{25}$

To rule out the influence of possible sensitizer aggregation, the PtOEP phosphorescence quantum yield $\left(\Phi_{\mathrm{Ph}}\right)$ was measured as a function of its concentration in PMMA (ESI Fig. S4 $\dagger$ ). $\Phi_{\mathrm{Ph}}$ remained indeed constant up to a PtOEP content of $0.3 \mathrm{wt} \%$, where we surmise aggregation starts to play a role. This finding indicates the absence of PtOEP aggregation in our films and points to FRET as the prime reason for emission quenching.

To quantify the FRET process, the singlet exciton diffusion length $\left(L_{\mathrm{D}}\right)$ in the upconverting films was evaluated and compared to the average distance needed for the upconverted singlets to reach sensitizer molecules. In the DPA/PtOEP/PMMA films containing $0.05 \mathrm{wt} \%$ of PtOEP and $25 \mathrm{wt} \%$ of DPA, the average distance $(d)$ between PtOEP molecules is estimated to be $\sim 14 \mathrm{~nm}$ (for a homogeneously dispersed film). Assuming that singlet excitons are generated via UC with equal probability in the entire volume of the film, $L_{\mathrm{D}}$ must be at least $1 / 2 \times d(=7$ $\mathrm{nm}$ ) for the FRET to be effective. In the upconverting films, $L_{\mathrm{D}}$ was evaluated via time-resolved fluorescence bulk-quenching measurements. ${ }^{36}$ For these measurements, films with an increasing concentration of quencher, phenyl-C61-butyric acid methyl ester (PCBM), were fabricated to estimate the relative fluorescence quenching efficiency and to determine the exciton diffusion by applying Stern-Volmer quenching analysis (see ESI Fig. S5 $\dagger){ }^{37}$ Two sets of films, i.e. with and without PtOEP, were prepared to specifically address the FRET from DPA to the sensitizer.

The results revealed a steady increase of $L_{\mathrm{D}}$ from 14 to $25 \mathrm{~nm}$ when the DPA concentration increased from 20 to $35 \mathrm{wt} \%$ in the absence of PtOEP (Fig. 3, squares). This behavior was found to be fully consistent with an enhanced exciton diffusivity on account of reduced intermolecular distances between DPA molecules. However, the values appear rather large taking into account that $L_{\mathrm{D}}$ of singlet excitons in amorphous organic films is typically below $10 \mathrm{~nm}$. The result can be explained on the basis of the work performed by Menke et al., ${ }^{38}$ who reported a substantial extension of $L_{\mathrm{D}}$ for chromophores that were diluted in a wide bandgap matrix. The dilution was shown to prolong fluorescence lifetime, increase $\Phi_{\mathrm{FL}}$, and enlarge spectral overlap. A similar situation is likely occurring in the upconverting films studied in this work, viz. melt-processing ensures good dispersion and minimizes concentration quenching, thus maintaining high $\Phi_{\mathrm{FL}}$ and long fluorescence lifetime.

Conversely, in films with PtOEP, $L_{\mathrm{D}}$ was almost independent of the DPA concentration and significantly shorter $(\sim 8 \mathrm{~nm})$ than that obtained for sensitizer-free films (Fig. 3, circles). Moreover, the estimated $L_{\mathrm{D}}$ value nearly corresponds to $1 / 2 \times d$, confirming that the singlet excitons can readily diffuse to the sensitizer molecules. This result further demonstrates that the PtOEP molecules act as singlet exciton traps in the upconverting films, thereby severely limiting their $L_{\mathrm{D}}$ and suppressing the intended radiative singlet decay in DPA. The findings validate the previously obtained $\Phi_{\mathrm{FL}}$ quenching amounting to $\sim 80 \%$ in DPA/ PMMA films in the presence of the sensitizer (Fig. 1a).

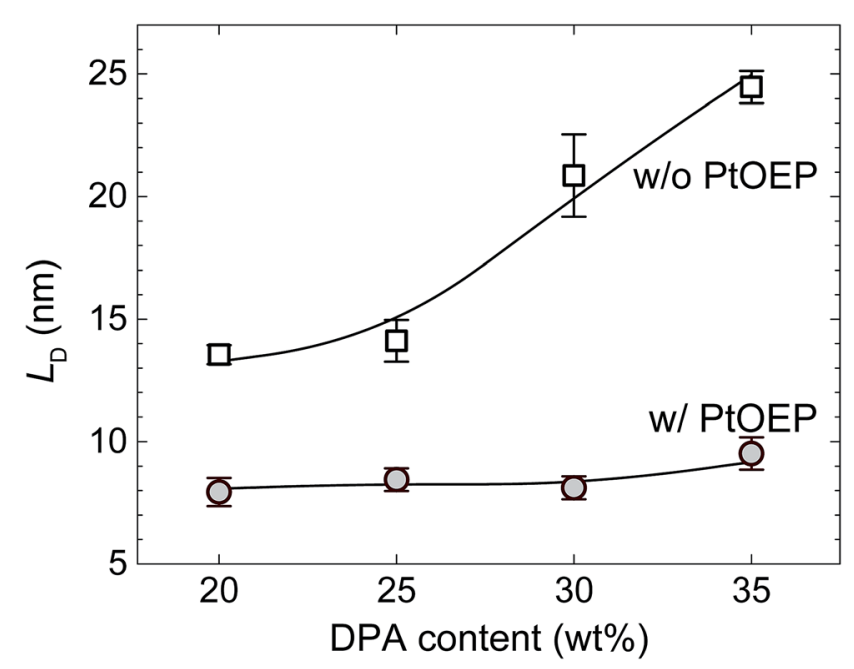

Fig. 3 Singlet exciton diffusion length as a function of DPA concentration in DPA/PMMA films with (circles, CPtOEP $=0.05 \mathrm{wt} \%$ ) and without (squares) PtOEP sensitizer. Lines are guides to the eye. 


\section{Singlet exciton sink approach}

Although a long triplet $L_{\mathrm{D}}$ is essential to ensure efficient TTA in rigid UC materials, a long singlet $L_{\mathrm{D}}$ is highly undesirable, as it can facilitate FRET to the sensitizer, and thus a reduction of $\Phi_{\mathrm{UC}}$. A straightforward way to avoid FRET is to reduce the sensitizer concentration (see Fig. 2). Nevertheless, it will inevitably limit light absorption thereby diminishing the generation of triplet states and ultimately the TTA-UC process. In principle, this problem can be solved by sensitizer-free single-component organic TTA-UC systems based on direct triplet absorption induced by heavy atom effect. ${ }^{39}$ While the general feasibility of this approach has been demonstrated, such systems display very low UC efficiencies, and the low molar absorptivity of the $\mathrm{S}-\mathrm{T}$ transition limits their usefulness.

Here, we explore another solution to circumvent the FRET issue accompanying large singlet $L_{\mathrm{D}}$ : a singlet exciton sink approach. The latter entails the intentional introduction of highly emissive exciton sinks, which trap singlets generated by TTA before they can even be quenched by a sensitizer molecule. Interestingly, Yanai and Kimizuka proposed a hypothetical, similar approach based on high-fluorescence-yield singlet energy collectors to improve the performance of TTA-UC systems through precisely controlled spatial organization. ${ }^{40}$ In contrast, our approach does not require a complex spatial distribution of the system components, but relies instead on homogeneously dispersed singlet sinks. This facile methodology is readily scalable and such materials were straightforwardly attained by the currently utilized melt-processing technique.

To be useful, the singlet sink must meet the following requirements: (i) display high $\Phi_{\mathrm{FL}}$ (desirably close to $100 \%$ ), (ii) have a singlet energy that is (slightly) lower than that of the emitter to ensure efficient FRET to the sink (note however that the energy should not be too low to preserve anti-Stokes emission), (iii) the singlet lifetime should be shorter than that of the emitter to ensure rapid emission from the sink, and (iv) the triplet energy must be higher than that of the emitter to avoid triplet exciton depopulation in the emitter.

The pyreneethynylene derivative (PE, 1,6-bis-[2,5di(dodecyloxyphenyl)ethynyl]pyrene, Fig. 4a) fulfills these requirements and was therefore chosen as a singlet sink for the DPA/PtOEP/PMMA blends presently investigated.$^{41} \mathrm{PE}$ exhibits a reasonably high $\Phi_{\mathrm{FL}}, 75 \%$ and $69 \%$ in dilute solution and melt-processed PMMA films, respectively, which is adequate for the demonstration of the approach. Moreover, the lowest energy and strongest absorption band of this compound at $435 \mathrm{~nm}$ overlaps well with the UC emission maximum of DPA (Fig. 4b). These conditions ensure efficient FRET from DPA to PE. The PE emission is redshifted by $\sim 12 \mathrm{~nm}$ relative to that of DPA, implying that the singlet energy is $\sim 75 \mathrm{meV}$ lower, which suffices to guarantee exciton trapping at PE sites at ambient and even elevated temperature.

The fluorescence decays of PE and DPA in PMMA films are compared in Fig. 4c. Following a mono-exponential decay, PE has a 6-fold shorter fluorescence lifetime ( $\sim 1.8 \mathrm{~ns})$ compared to DPA, which ensures much faster radiative relaxation of the trapped excitons and lower probability for their accumulation at the sink sites or back-transfer to DPA.

The triplet energy of $\mathrm{PE}$, as determined from the onset of phosphorescence spectrum (ESI Fig. S7 $\dagger$ ), is $1.97 \mathrm{eV}$, which is $\sim 160 \mathrm{meV}$ higher than that of DPA. ${ }^{42}$ This is important as it prevents triplet exciton transfer from DPA to PE. The energy level alignment of DPA/PtOEP system with added singlet sink $\mathrm{PE}$ is illustrated in the inset of Fig. $4 \mathrm{~b}$.

At first glance, the energetics regarding singlet sink may seem rather stringent. For the efficient FRET to the sink its singlet energy must to be lower than that of the emitter to ensure good spectral overlap between sink absorption and emitter emission. On the other hand, sink emission must not be to be too low in order to avoid absorption by the sensitizer's $Q$ band. In part, this problem could be solved by utilizing sensitizers with lower Q band (larger energy separation between Soret- and Q-band). E.g. for NIR range, sensitizers based on metallonaphthalocyanines could be suitable. ${ }^{43}$ They have larger transparency window $(400-700 \mathrm{~nm})$ as compared to that of PtOEP (400-500 nm). On the other hand, finding a highlyemissive sink with desired energy levels should not be a big issue. A vast number of fluorophores (with $\Phi_{\mathrm{FL}}$ close to $100 \%$ ) of desired wavelength with typically narrow absorption/emission bands and small Stokes shift are widely available. Semiconductor quantum dots, which emission/absorption wavelength can be finely tuned by varying their size, could be an attractive alternative to organic singlet sink compounds.

To verify the proposed strategy of singlet exciton trapping, the best performing DPA/PtOEP/PMMA mixture (containing $25 \mathrm{wt} \%$ DPA and $0.01 \mathrm{wt} \%$ PtOEP) was used to create blends with PE. The PE concentration was varied from 0.001 to $1 \mathrm{wt} \%$. For these compositions, $\Phi_{\mathrm{UC}}$ is shown as a function of $\mathrm{PE}$ concentration in Fig. 5. At PE concentrations below $2 \times$ $10^{-3} \mathrm{wt} \%$, almost no impact of the sink on $\Phi_{\mathrm{UC}}$ is noticed. However, when the PE content is increased to $0.01 \mathrm{wt} \%$, a 1.5fold enhancement of $\Phi_{\mathrm{UC}}$ and a maximum value of $2.7 \%$ is observed. Beyond this point $\Phi_{\mathrm{UC}}$ gradually decreased, perhaps due to PE aggregation and partially due to increased FRET from $\mathrm{PE}$ to PtOEP (the Q bands of PtOEP overlap rather well with PE emission). Nevertheless, maximal $\Phi_{\mathrm{UC}}$ of $2.7 \%$ achieved via singlet sink approach is among the highest $\Phi_{\mathrm{UC}}$ reported so far in rigid amorphous solids. ${ }^{21-23}$

To confirm that the FRET from PE to PtOEP in turn is responsible for $\Phi_{\mathrm{UC}}$ quenching observed at PE concentrations > $0.01 \mathrm{wt} \%$, we have estimated and compared FRET rates for the $\mathrm{DPA} \rightarrow \mathrm{PtOEP}, \mathrm{DPA} \rightarrow \mathrm{PE}, \mathrm{PE} \rightarrow \mathrm{DPA}$ and $\mathrm{PE} \rightarrow$ PtOEP processes at the fixed DPA and PtOEP concentrations of $25 \mathrm{wt} \%$ and $0.01 \mathrm{wt} \%$, respectively (see ESI Table S2 and Fig. S6†). From this data it is clear that at low singlet sink (PE) concentrations excitons trapped by PE will eventually emit since FRET rates for $\mathrm{PE} \rightarrow$ PtOEP are well below natural decay rate of PE $\left(5 \times 10^{8}\right.$ $\left.\mathrm{s}^{-1}\right)$. Before emission excitons can undergo numerous FRET cycles between PE and DPA due to the large transfer $\left(\sim 10^{13} \mathrm{~s}^{-1}\right)$ and back-transfer $\left(\sim 3 \times 10^{10} \mathrm{~s}^{-1}\right)$ rates. With PE concentration well exceeding $0.01 \mathrm{wt} \%$, FRET rate for PE $\rightarrow$ PtOEP becomes comparable to $\mathrm{PE}$ decay rate and thus can contribute to the observed $\Phi_{\mathrm{UC}}$ quenching. 
(a)

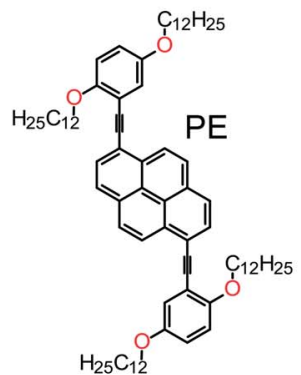
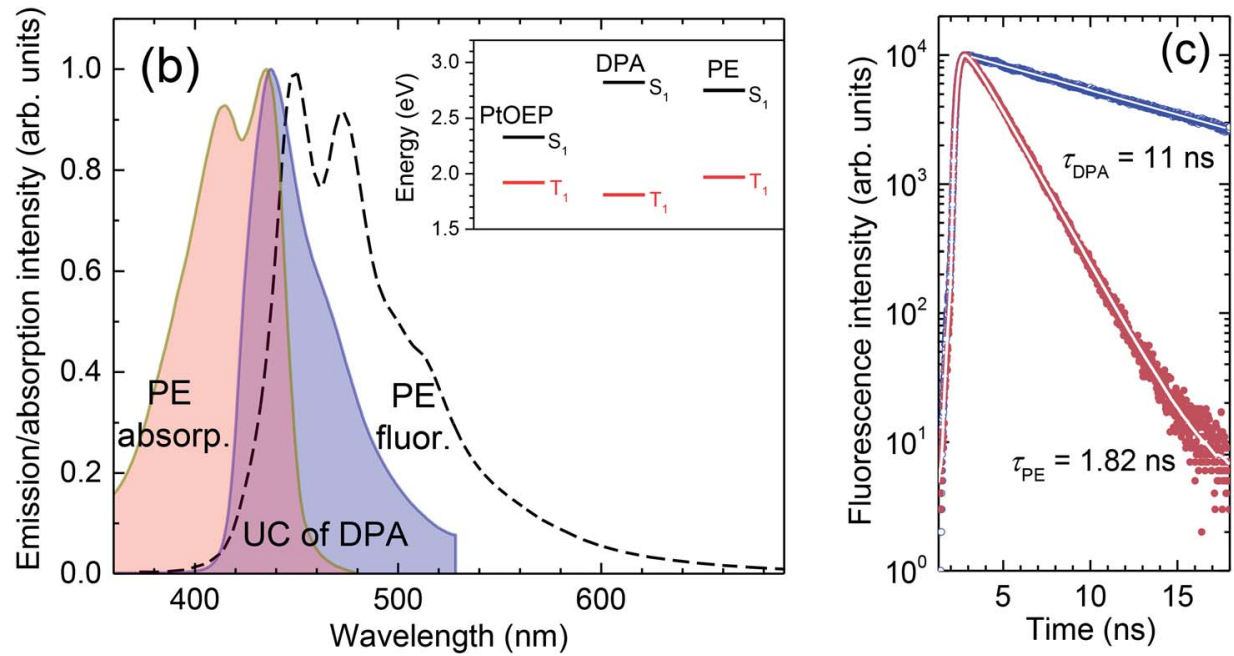

Fig. 4 (a) Chemical structure of the singlet exciton sink PE. (b) Normalized absorption and fluorescence spectra of the PE in PMMA ( $C_{P E}=$ $0.01 \mathrm{wt} \%$ ) and the UC emission spectrum of DPA/PtOEP/PMMA ( $C_{\text {DPA }}=25 \mathrm{wt} \%, C_{\text {PtOEP }}=0.01 \mathrm{wt} \%$ ). The inset shows energy level alignment of DPA/PtOEP system with added singlet sink PE. (c) Fluorescence decays of PE (red points) and DPA (blue points) in PMMA ( $c_{P E}=0.01$ wt $\%, c_{D P A}=$ 25 wt\%). Lines are single exponential fits.

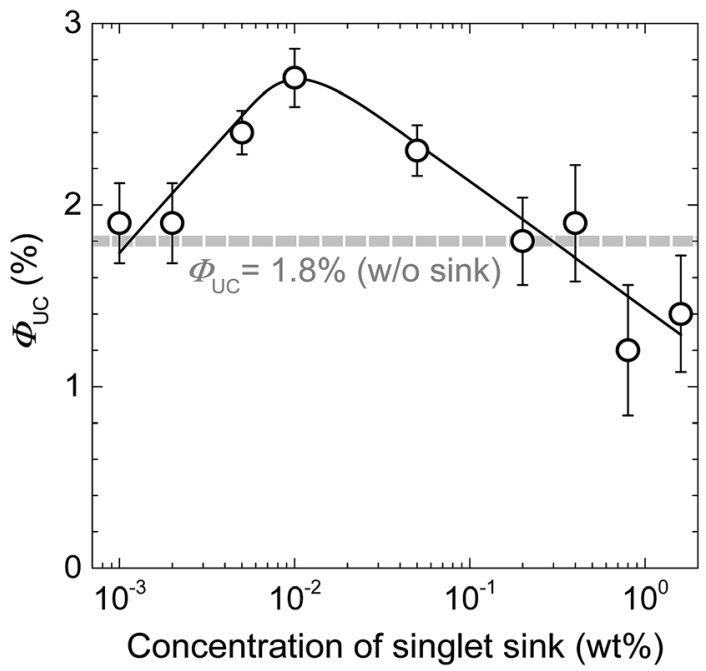

Fig. 5 UC quantum yield of DPA/PtOEP/PE singlet sink/PMMA films $\left(c_{\text {DPA }}=25 \mathrm{wt} \%, c_{\text {PtOEP }}=0.01 \mathrm{wt} \%\right)$ as a function of singlet sink concentration. The solid line is a guide to the eye. The dashed line indicates the maximum $\Phi_{\cup \mathrm{C}}$ of the corresponding material without the singlet sink. The error bars were derived by averaging data of three samples.

Theoretically, since PtOEP $\left(c_{\mathrm{PtOEP}}=0.01 \mathrm{wt} \%\right)$ reduces $\Phi_{\mathrm{FL}}$ of DPA by a factor of 2.5 , from $75 \%$ to $30 \%$ (Fig. 2), a maximal enhancement of $\Phi_{\mathrm{UC}}$ of up to $4.5 \%(1.8 \% \times 2.5)$ should be attainable if all DPA singlets were collected by PE. The fact that a smaller enhancement of $\Phi_{\mathrm{UC}}$ is observed indicates that exciton trapping by the sink is incomplete.

A typical UC emission spectrum of the films with $0.01 \mathrm{wt} \%$ of PE is shown in Fig. 6. To elucidate its origin, the spectrum was superimposed with that obtained from the films without singlet sink and also with the fluorescence spectrum of a film of PE in PMMA. The comparison of these spectra clearly shows that the
UC emission originates from both, the DPA and PE emitters. The quantitative spectral analysis (based on integrated intensities) reveals that the UC emission is composed of $\sim 2 / 3$ DPA and $\sim 1 / 3$ PE emission. This result confirms again that the collection of singlets by the sink is not fully effective.

Taking into account that at PE concentration of $0.01 \mathrm{wt} \%$ FRET rate for PE $\rightarrow$ PtOEP is negligible as compared to the decay rate of excitons in PE, it is plausible to assume that all the exciton trapped by the sink will contribute to the emission. In other words, for the $1 / 3$ part of UC emission associated with PE we can expect $\Phi_{\mathrm{UC}}$ to attain its maximum value, i.e. $4.5 \%$ (= $1.8 \% \times 2.5$ ), whereas for the rest $2 / 3$ DPA-related part of UC emission only $1.8 \%$ as this part is subjected to quenching by

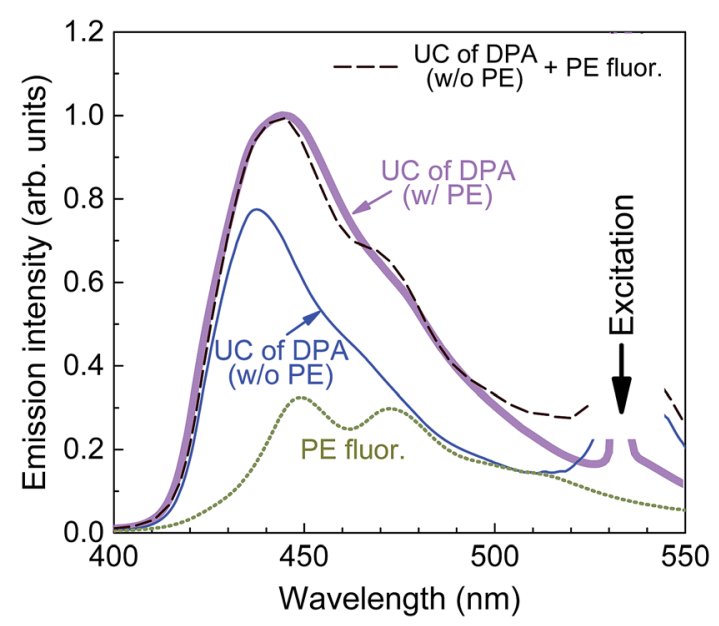

Fig. 6 Fluorescence spectrum (dotted line) of the singlet sink $P E$ in PMMA $\left(c_{\mathrm{PE}}=0.01 \mathrm{wt} \%\right), \mathrm{UC}$ spectra of DPA/PtOEP/PMMA $\left(c_{\mathrm{DPA}}=\right.$ $25 \mathrm{wt} \%, C_{\text {PtOEP }}=0.01 \mathrm{wt} \%$ ) without (thin line) and with (thick line) PE $\left(c_{\mathrm{PE}}=0.01 \mathrm{wt} \%\right)$, and a sum of the first two spectra (dashed line) at a ratio of $1 / 3$ and $2 / 3$, respectively. 
PtOEP. Indeed this is confirmed by the sum of $\Phi_{\mathrm{UC}}$ of both parts $1 / 3 \times 4.5 \%$ and $2 / 3 \times 1.8 \%$, which is equal to the overall $\Phi_{\mathrm{UC}}$ of DPA/PtOEP/PE/PMMA film (2.7\%). This result indicates that there are no significant losses once transfer to $\mathrm{PE}$ is realized and the most important limitation in this system is DPA $\rightarrow$ PE transfer.

A plausible way to enhance the collection efficiency is to increase the sink concentration while avoiding its aggregation. Alternatively, the sink can be tethered to the DPA emitter in order to enhance efficient transfer.

In the DPA/PtOEP/PMMA films containing the PE singlet sink, the excitation density dependence of the UC emission intensity exhibited a typical quadratic behavior at lower excitation densities, which changed to linear at a threshold intensity $\left(I_{\text {th }}\right)$ at higher power densities (ESI Fig. S8 $\left.\dagger\right) \cdot{ }^{\mathbf{4 4 , 4 5}} I_{\text {th }}$ indicates the dominance of the TTA process over other decay pathways. It was

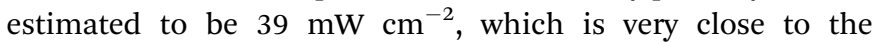
threshold for the best performing DPA/PtOEP/PMMA films (without the sink).$^{20}$ Importantly, this invariance signifies that the singlet sink influences neither the TTA, nor the triplet exciton manifold of DPA, and only affects upconverted singlets (vide supra). Specifically, the involvement of PE triplet states would otherwise manifest itself through a reduced concentration of DPA triplets and a significant increase of $I_{\mathrm{th}}$.

The singlet sink approach was successfully implemented to mitigate UC emission quenching by FRET in a DPA/PtOEP model system and should be readily generalizable to other solid state UC systems, provided that an adequate singlet sink meeting the aforementioned conditions be identified for each system. Certainly, suppressed detrimental energy transfer via introduction of a singlet sink implies some tradeoff between enhancing TTA-UC efficiency and reducing the energy of the harvested state. Therefore from a TTA-UC solar cell perspective, depending on the particular absorber used, a proper sensitizeremitter and sink combination needs to be chosen to achieve the final improvement in photoconversion efficiency of the cell.

\section{Conclusions}

In summary, this work addressed the serious FRET issue limiting radiative decay of the singlet emitter excitons (produced via TTA) through their back-transfer to the sensitizer. This issue is a challenging problem especially for nextgeneration sensitizers, such as semiconductor nanocrystals and direct triplet absorbers, which lack transparency window for UC emission. It causes substantial UC losses even in the well-studied DPA/PtOEP model system designed to reduce overlap of DPA emission and PtOEP absorption. Backed up by quantitative Stern-Volmer analysis of singlet exciton diffusion in highly DPA-loaded (15-40 wt \%) rigid UC films, FRET induced quenching can decrease $\Phi_{\mathrm{FL}}$ of DPA to $1 / 6^{\text {th }}$ of its original value for PtOEP concentrations as low as $0.05 \mathrm{wt} \%$. To suppress FRETassisted singlet exciton quenching by sensitizer molecules, we implemented a singlet exciton sink approach, which necessitated an alternative singlet transfer route to intentionally introduced, highly emissive traps (sinks). The utilization of a pyreneethynylene derivative as the sink for the current solid- state UC system confirmed the validity of our methodology and exhibited very promising results leading to a 1.5-fold increase of $\Phi_{\mathrm{UC}}$, while keeping $I_{\text {th }}$ almost unchanged. Although quantitative productive harvesting of upconverted singlets by intentionally incorporating singlet sinks could have improved $\Phi_{\mathrm{UC}}$ up to 2.5 times (up to $4.5 \%$ ), the achieved maximal $\Phi_{\mathrm{UC}}$ of $2.7 \%$ remains one of the highest reported so far for rigid amorphous sensitized UC systems. These results obtained for DPA/PtOEP system are extremely encouraging. They suggest that the singlet sink approach can be extended to other UC systems suffering from FRET issues and open the door to a variety of novel energy transfer strategies to increase the efficiency and therefore the usefulness of upconverting systems.

\section{Conflicts of interest}

There are no conflicts to declare.

\section{Acknowledgements}

The research at Vilnius University was funded by a grant (No. SMIP-17-77) from the Research Council of Lithuania.

\section{References}

1 O. S. Kwon, H. S. Song, J. Conde, H. Kim, N. Artzi and J.-H. Kim, ACS Nano, 2016, 10, 1512-1521.

2 R. S. Khnayzer, J. Blumhoff, J. A. Harrington, A. Haefele, F. Deng and F. N. Castellano, Chem. Commun., 2012, 48, 209-211.

3 M. Borisov Sergey, L. Christoph and K. Ingo, Adv. Funct. Mater., 2012, 22, 4360-4368.

4 W. Wang, Q. Liu, C. Zhan, A. Barhoumi, T. Yang, R. G. Wylie, P. A. Armstrong and D. S. Kohane, Nano Lett., 2015, 15, 63326338.

5 S. H. C. Askes, M. S. Meijer, T. Bouwens, I. Landman and S. Bonnet, Molecules, 2016, 21, 1460.

6 S. Baluschev, T. Miteva, V. Yakutkin, G. Nelles, A. Yasuda and G. Wegner, Phys. Rev. Lett., 2006, 97, 143903.

7 V. Gray, D. Dzebo, M. Abrahamsson, B. Albinsson and K. Moth-Poulsen, Phys. Chem. Chem. Phys., 2014, 16, 10345-10352.

8 T. F. Schulze and T. W. Schmidt, Energy Environ. Sci., 2015, 8, 103-125.

9 Y. Y. Cheng, A. Nattestad, T. F. Schulze, R. W. MacQueen, B. Fückel, K. Lips, G. G. Wallace, T. Khoury, M. J. Crossley and T. W. Schmidt, Chem. Sci., 2015, 7, 559-568.

10 L. Frazer, J. K. Gallaher and T. W. Schmidt, ACS Energy Lett., 2017, 2, 1346-1354.

11 A. Monguzzi, R. Tubino, S. Hoseinkhani, M. Campione and F. Meinardi, Phys. Chem. Chem. Phys., 2012, 14, 4322-4332.

12 Y. C. Simon and C. Weder, J. Mater. Chem., 2012, 22, 2081720830.

13 T. Ogawa, N. Yanai, A. Monguzzi and N. Kimizuka, Sci. Rep., 2015, 5, 10882.

14 R. Vadrucci, A. Monguzzi, F. Saenz, B. D. Wilts, Y. C. Simon and C. Weder, Adv. Mater., 2017, 29, 1702992. 
15 A. Monguzzi, F. Bianchi, A. Bianchi, M. Mauri, R. Simonutti, R. Ruffo, R. Tubino and F. Meinardi, Adv. Energy Mater., 2013, 3, 680-686.

16 R. Vadrucci, C. Weder and Y. C. Simon, Mater. Horiz., 2014, 2, 120-124.

17 P. Duan, N. Yanai, H. Nagatomi and N. Kimizuka, J. Am. Chem. Soc., 2015, 137, 1887-1894.

18 D. C. Thévenaz, A. Monguzzi, D. Vanhecke, R. Vadrucci, F. Meinardi, Y. C. Simon and C. Weder, Mater. Horiz., 2016, 3, 602-607.

19 P. B. Merkel and J. P. Dinnocenzo, J. Lumin., 2009, 129, 303306.

20 S. Raišys, K. Kazlauskas, S. Juršènas and Y. C. Simon, ACS Appl. Mater. Interfaces, 2016, 8, 15732-15740.

21 A. Turshatov, D. Busko, N. Kiseleva, S. L. Grage, I. A. Howard and B. S. Richards, ACS Appl. Mater. Interfaces, 2017, 9, 82808286.

22 A. Monguzzi, M. Mauri, M. Frigoli, J. Pedrini, R. Simonutti, C. Larpent, G. Vaccaro, M. Sassi and F. Meinardi, J. Phys. Chem. Lett., 2016, 7, 2779-2785.

23 A. Monguzzi, M. Frigoli, C. Larpent, R. Tubino and F. Meinardi, Adv. Funct. Mater., 2012, 22, 139-143.

24 M. Wu, D. N. Congreve, M. W. B. Wilson, J. Jean, N. Geva, M. Welborn, T. Van Voorhis, V. Bulović, M. G. Bawendi and M. A. Baldo, Nat. Photonics, 2016, 10, 31-34.

25 V. Gray, K. Moth-Poulsen, B. Albinsson and M. Abrahamsson, Coord. Chem. Rev., 2018, 362, 54-71.

26 T. N. Singh-Rachford and F. N. Castellano, Coord. Chem. Rev., 2010, 254, 2560-2573.

27 X. Li and M. L. Tang, Chem. Commun., 2017, 53, 4429-4440.

28 S. P. Hill, T. Banerjee, T. Dilbeck and K. Hanson, J. Phys. Chem. Lett., 2015, 6, 4510-4517.

29 N. Yanai and N. Kimizuka, Acc. Chem. Res., 2017, 50, 24872495.
30 V. Gray, K. Börjesson, D. Dzebo, M. Abrahamsson, B. Albinsson and K. Moth-Poulsen, J. Phys. Chem. C, 2016, 120, 19018-19026.

31 P. E. Keivanidis, S. Baluschev, G. Lieser and G. Wegner, ChemPhysChem, 2009, 10, 2316-2326.

32 T. C. Wu, D. N. Congreve and M. A. Baldo, Appl. Phys. Lett., 2015, 107, 031103.

33 N. Yanai, M. Kozue, S. Amemori, R. Kabe, C. Adachi and N. Kimizuka, J. Mater. Chem. C, 2016, 4, 6447-6451.

34 Y. Sasaki, S. Amemori, H. Kouno, N. Yanai and N. Kimizuka, J. Mater. Chem. C, 2017, 5, 5063-5067.

35 S. H. Lee, J. R. Lott, Y. C. Simon and C. Weder, J. Mater. Chem. C, 2013, 1, 5142-5148.

36 J. D. A. Lin, O. V. Mikhnenko, J. Chen, Z. Masri, A. Ruseckas, A. Mikhailovsky, R. P. Raab, J. Liu, P. W. M. Blom, M. A. Loi, C. J. García-Cervera, I. D. W. Samuel and T.-Q. Nguyen, Mater. Horiz., 2014, 1, 280-285.

37 H.-Y. Hsu, J. H. Vella, J. D. Myers, J. Xue and K. S. Schanze, J. Phys. Chem. C, 2014, 118, 24282-24289.

38 S. M. Menke, W. A. Luhman and R. J. Holmes, Nat. Mater., 2013, 12, 152-157.

39 K. Okumura, M. Matsuki, T. Yamada, N. Yanai and N. Kimizuka, ChemistrySelect, 2017, 2, 7597-7601.

40 N. Yanai and N. Kimizuka, Chem. Commun., 2016, 52, 53545370.

41 J. Gupta, S. Vadukumpully and S. Valiyaveettil, Polymer, 2010, 51, 5078-5086.

42 H. Goudarzi and P. E. Keivanidis, ACS Appl. Mater. Interfaces, 2017, 9, 845-857.

43 S. Amemori, N. Yanai and N. Kimizuka, Phys. Chem. Chem. Phys., 2015, 17, 22557-22560.

44 A. Haefele, J. Blumhoff, R. S. Khnayzer and F. N. Castellano, J. Phys. Chem. Lett., 2012, 3, 299-303.

45 A. Monguzzi, J. Mezyk, F. Scotognella, R. Tubino and F. Meinardi, Phys. Rev. B, 2008, 78, 195112. 